ARTICLE OPEN

\title{
Removal of nonimpacted third molars alters the periodontal condition of their neighbors clinically, immunologically, and microbiologically
}

\author{
Yi Tian ${ }^{1}$, Lijuan Sun ${ }^{1}$, Honglei Qu ${ }^{1}$, Yang Yang ${ }^{1}$ and Faming Chen (D)
}

\begin{abstract}
Considering the adverse effects of nonimpacted third molars (N-M3s) on the periodontal health of adjacent second molars (M2s), the removal of $\mathrm{N}-\mathrm{M} 3 \mathrm{~s}$ may be beneficial to the periodontal health of their neighbors. This study aimed to investigate the clinical, immunological, and microbiological changes of the periodontal condition around $\mathrm{M} 2 \mathrm{~s}$ following removal of neighboring $\mathrm{N}-\mathrm{M} 3 \mathrm{~s}$ across a 6-month period. Subjects with at least one quadrant containing an intact first molar (M1), M2, and N-M3 were screened and those who met the inclusion criteria and decided to receive N-M3 extraction were recruited in the following investigation. $\mathrm{M} 2$ periodontal condition was interrogated before $M 3$ extraction (baseline) and at 3 and 6 months postoperatively. Improvements in clinical periodontal indexes of $\mathrm{M} 2 \mathrm{~s}$ in response to their adjacent N-M3 removal, along with changes in inflammatory biomarkers among gingival crevicular fluid (GCF) and the composition of subgingival plaque collected from the distal sites of the M2s of the targeted quadrant were parallelly analyzed. Complete data of 26 tooth extraction patients across the follow-up period were successfully obtained and subsequently applied for statistical analysis. Compared to the baseline, the periodontal condition of M2s was significantly changed 6 months after N-M3 removal; specifically, the probing depth of M2s significantly reduced $(P<0.001)$, the matrix metalloproteinase (MMP)-8 concentration involved in GCF significantly decreased $(P=0.025)$, and the abundance of the pathogenic genera unidentified Prevotellaceae and Streptococcus significantly decreased $(P<0.001$ and $P=0.009$, respectively). We concluded that N-M3 removal was associated with superior clinical indexes, decreased GCF inflammatory biomarkers, and reduced pathogenic microbiome distribution within the subgingival plaque. Although the retention or removal of N-M3s continues to be controversial, our findings provide additional evidence that medical decisions should be made as early as possible or at least before the neighboring teeth are irretrievably damaged.
\end{abstract}

International Journal of Oral Science (2021)13:5

; https://doi.org/10.1038/s41368-020-00108-y

\section{INTRODUCTION}

Third molar (M3) related diseases (e.g., pericoronitis, caries, odontogenic cysts, and tumors), especially caries, root resorption, and periodontal diseases of their adjacent teeth, have received extensive attention from patients and dentists. ${ }^{1,2}$ Although M3associated diseases are more common in the clinic when M3s are in an impacted positioning (impacted M3s, I-M3s), ${ }^{3,4}$ recent evidence suggests that even nonimpacted M3s (N-M3s) may cause severe periodontal damage to their adjacent second molars (M2s). ${ }^{3,5,6}$

In this context, a longitudinal study over 25 years found that in healthy American males, the retention of M3s, even if they were asymptomatic, increased the risk of periodontal destruction of adjacent M2s compared to that of those without M3s. ${ }^{3}$ Clearly, NM3s had not been excluded from the investigation in this study. Regarding the adverse effects of N-M3s, a German investigation reported that in the total population, M2s had a 1.45 -fold odds ratio of increased probing depth (PD) in the presence of adjacent $\mathrm{N}-\mathrm{M} 3 \mathrm{~s}$ compared to the absence of $\mathrm{M} 3 \mathrm{~s}^{5}{ }^{5}$ Similar conclusions based on clinical studies were also reported in Chinese patients. ${ }^{7-9}$ Based on orthopantomograms of 1958 patients, our previous investigation also found that $\mathrm{M} 2 \mathrm{~s}$ with neighboring $\mathrm{N}-\mathrm{M} 3 \mathrm{~s}$ faced a 1.77-fold increased risk of alveolar bone resorption when compared to those without adjacent $\mathrm{M} 3 \mathrm{~s}^{6}{ }^{6}$ Interrogating the negative influences of $\mathrm{N}-\mathrm{M} 3 \mathrm{~s}$ from another angle, researchers also demonstrated that the removal of M3s including but not limited to $\mathrm{N}-\mathrm{M} 3 \mathrm{~s}$ led to improved periodontal conditions for their neighboring $\mathrm{M} 2 \mathrm{~s} .{ }^{10,11}$

The investigations mentioned above suggest that the effects of $\mathrm{N}-\mathrm{M} 3$ retention on their neighboring teeth should receive more attention from both patients and dentists. Considering the difficulty of treating irreversible periodontal destruction, such as alveolar bone resorption, preventing the occurrence of periodontitis is of key importance. Since periodontal diseases were initiated by the colonization of subgingival periodontal pathogens and corresponding changes in the inflammatory-immune system, ${ }^{12}$ the negative impacts of N-M3s on their neighbors are likely to result from the interplay between the subgingival microbiome and host response across the local microenvironment of the periodontium. In this regard, it is reasonable to speculate that the removal of $\mathrm{N}-\mathrm{M} 3 \mathrm{~s}$ should be able to positively alter the local immunological and microbiological conditions around their adjacent M2s.

\footnotetext{
${ }^{1}$ National Clinical Research Center for Oral Diseases, Department of Periodontology, School of Stomatology, Fourth Military Medical University, Xi'an, China Correspondence: Faming Chen (cfmsunhh@fmmu.edu.cn)

These authors contributed equally: Yi Tian, Lijuan Sun
}

Received: 15 August 2020 Revised: 17 October 2020 Accepted: 26 November 2020

Published online: 07 February 2021 
In this study, the clinical, immunological, and microbiological changes in the periodontal condition around $\mathrm{M} 2 \mathrm{~s}$ in terms of clinical periodontal indexes, inflammatory biomarkers in gingival crevicular fluid (GCF), and the composition of subgingival plaque of $\mathrm{M} 2 \mathrm{~s}$ were investigated following removal of their neighboring $\mathrm{N}-\mathrm{M} 3 \mathrm{~s}$ across a 6-month period. The specific aim of the study was to identify how N-M3 removal alters the periodontal condition of their neighbors and to provide additional evidence that N-M3s lead to adverse effects on the periodontal health of adjacent M2s.

\section{RESULTS}

Subjects enrolled

From May 2019 to June 2019, 121 subjects were recorded in the $\mathrm{N}-\mathrm{M} 3 \mathrm{~s}(+)$ group and 54 in the M3s (-) group at the prescreening stage. Based on inclusion and exclusion criteria and/or the agreement of tooth extraction, only 30 of the N-M3s (+) and 26 of the M3s (-) subjects were included in the longitudinal study (with one included quadrant per patient, randomly selected if multiple quadrants were eligible); 4 participants were lost or excluded during the 6-month longitudinal observation stage. After a 6-month follow-up, complete data were successfully obtained from 26 participants in the N-M3s (+) group and 28 participants in the M3s (-) group, and those data were applied for the subsequent statistical analysis (Fig. 1).

Sociodemographic information and clinical periodontal indexes of the participants

The demographic characteristics and locations of target quadrants of the $26 \mathrm{~N}-\mathrm{M} 3 \mathrm{~s}(+)$ and $28 \mathrm{M} 3 \mathrm{~s}(-)$ participants were homogeneous between groups $(P>0.05$, Table 1). Clinical periodontal indexes of the targeted $\mathrm{M} 2 \mathrm{~s}$ obtained at the baseline (T0) and across the follow-up period ( 3 months postoperatively was recorded as T1, while 6 months postoperatively was recorded as T2) are presented in Table 2. Compared to the baseline measurement (T0) in the N-M3s (+) group, PD was significantly reduced 6 months (T2) after the removal of the neighboring $\mathrm{N}-\mathrm{M} 3 \mathrm{~s}(P<0.001)$, while changes in the plaque index $(\mathrm{PLI})$, the prevalence of at least three sites that bled after probing $(\mathrm{BOP}+)$, at least one probing site $\geq 5 \mathrm{~mm}(\mathrm{PD} 5+)$, and
GCF volume showed no statistical significance among T0-T2 $(P=0.280, P=0.307, P=0.247$, and $P=0.857$, respectively). When the M3s (-) group was used as the control, significantly

Table 1. Demographic characteristics of participants $(N=54)$ and locations of targeted quadrants

\begin{tabular}{|c|c|c|c|c|}
\hline Characteristics & $\begin{array}{l}\text { All } \\
(N=54)\end{array}$ & $\begin{array}{l}\text { N-M3s }(+) \\
(N=26)\end{array}$ & $\begin{array}{l}\text { M3s (-) } \\
(N=28)\end{array}$ & $P$ \\
\hline \multicolumn{5}{|l|}{ Age/years } \\
\hline Mean \pm SD & $25.9 \pm 7.4$ & $24.9 \pm 6.2$ & $26.8 \pm 8.4$ & 0.361 \\
\hline Range & $18-56$ & $18-48$ & $18-56$ & \\
\hline \multicolumn{5}{|l|}{ Gender/\% } \\
\hline Male & 53.7 & 40.7 & 60.7 & 0.284 \\
\hline Female & 46.3 & 59.3 & 39.3 & \\
\hline \multicolumn{5}{|l|}{ Ethnicity/\% } \\
\hline Han Chinese & 98.1 & 96.3 & 100.0 & 0.481 \\
\hline Other & 1.9 & 3.7 & 0.0 & \\
\hline \multicolumn{5}{|l|}{ Education level/\% } \\
\hline College or above & 83.3 & 88.5 & 78.6 & 0.470 \\
\hline High school or below & 16.7 & 11.5 & 21.4 & \\
\hline \multicolumn{5}{|c|}{ Dental scaling frequency/\% } \\
\hline $\begin{array}{l}\text { At least once every } \\
2 \text { years }\end{array}$ & 24.1 & 23.1 & 25.0 & 0.869 \\
\hline Never & 75.9 & 76.9 & 75.0 & \\
\hline \multicolumn{5}{|l|}{ Dental visit frequency/\% } \\
\hline $\begin{array}{l}\text { At least once every } \\
2 \text { years }\end{array}$ & 37.0 & 35.7 & 35.7 & 0.700 \\
\hline Only when necessary & 63.0 & 64.3 & 64.3 & \\
\hline \multicolumn{5}{|c|}{ Locations of targeted quadrants $/ \%$} \\
\hline Maxillary & 87.0 & 96.2 & 78.6 & 0.102 \\
\hline Mandibular & 13.0 & 3.8 & 21.4 & \\
\hline
\end{tabular}

Note: N-M3s $(+), \mathrm{M} 2 \mathrm{~s}$ with neighboring nonimpacted third molars; M3s (-), M2s without neighboring third molars; SD standard deviation.

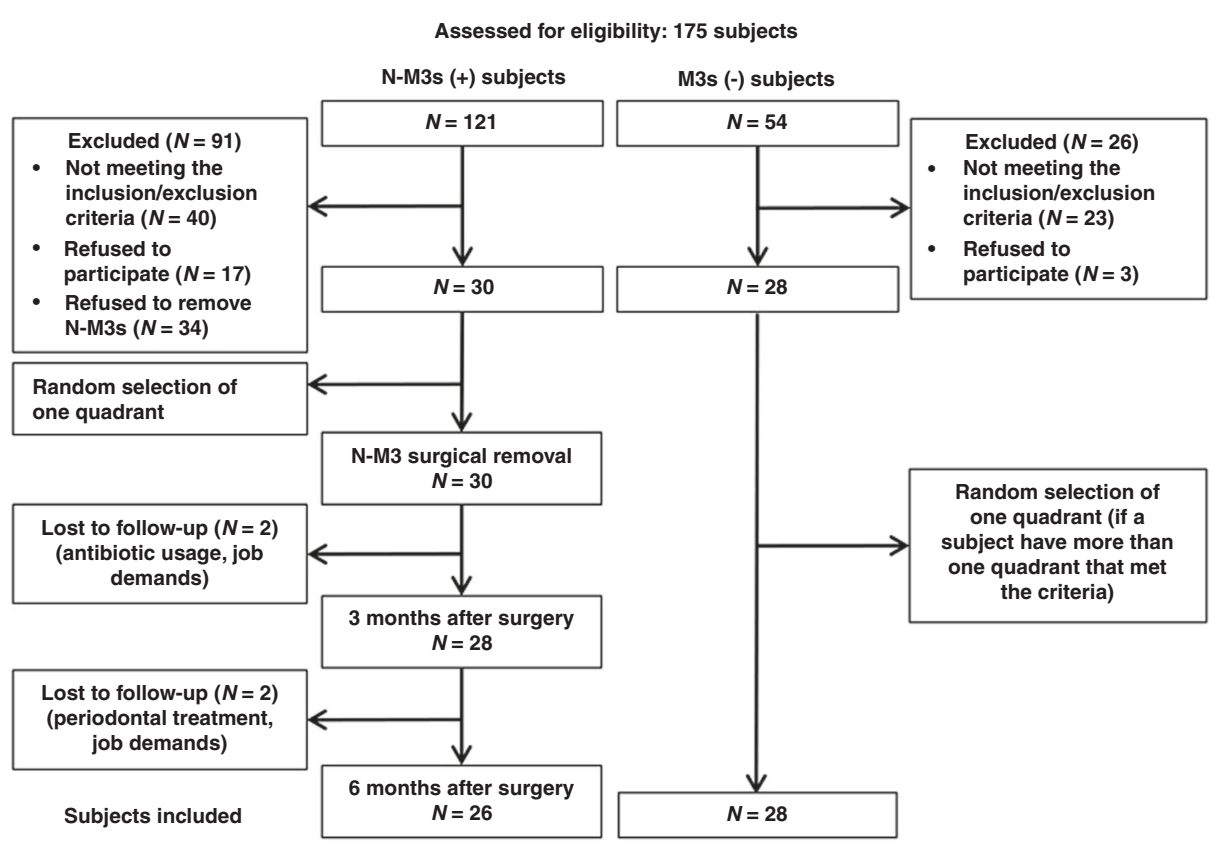

Fig. 1 Flow chart of the study design. $N$, number of patients; N-M3s $(+)$, second molars (M2s) with neighboring nonimpacted third molars; M3s (-), M2s without neighboring third molars 


\begin{tabular}{|c|c|c|c|c|c|c|c|}
\hline Groups & & Teeth (M2s) & $\mathrm{PD} / \mathrm{mm}($ mean $\pm \mathrm{SD})$ & $\mathrm{PLI}($ mean $\pm \mathrm{SD})$ & PD5 $+/ \%$ & $\mathrm{BOP}+/ \%$ & $\mathrm{GCF} / \mu \mathrm{L}($ mean $\pm \mathrm{SD})$ \\
\hline & $P^{a}$ & & $<0.001$ & 0.150 & 0.184 & 0.006 & 0.022 \\
\hline & $\mathrm{T} 1$ & 26 & $2.53 \pm 0.32$ & $1.88 \pm 0.59$ & 3.8 & 23.1 & $1.23 \pm 0.37$ \\
\hline & $\mathrm{T} 2$ & 26 & $2.48 \pm 0.23$ & $1.98 \pm 0.54$ & 7.7 & 34.6 & $1.23 \pm 0.46$ \\
\hline & $P^{a}$ & & 0.685 & 0.948 & 0.604 & 0.012 & 0.035 \\
\hline & $P^{2}$ & & $<0.001$ & NS & NS & NS & NS \\
\hline \multicolumn{2}{|l|}{ M3s (-) } & 28 & $2.49 \pm 0.30$ & $1.95 \pm 0.50$ & 3.6 & 7.1 & $0.97 \pm 0.37$ \\
\hline \multicolumn{8}{|c|}{$\begin{array}{l}-\mathrm{M} 3 \text { removal; } \mathrm{T} 2 \\
\mathrm{~N}-\mathrm{M} 3 \mathrm{~s}(-) \text { and } \mathrm{N} \\
\text { arisons by the } \mathrm{Bc} \\
\text { change among } \mathrm{T}\end{array}$} \\
\hline
\end{tabular}

increased PD, an elevated prevalence of $\mathrm{BOP}+$, and significantly increased GCF volume were recorded before N-M3 extraction (T0) $(P<0.001, P=0.006$, and $P=0.022$, respectively), but only $P D$ reached a similar level to that of the control 6 months postoperatively (T2) $(P=0.685)$, while the prevalence of $\mathrm{BOP}+$ and the GCF volume remained greater than those of the control $(P=0.012$ and $P=0.035$, respectively). To identify the potential influence of $\mathrm{N}-\mathrm{M} 3 \mathrm{~s}$ on tissue damage of their neighbors, we compared the mean PD of distobuccal and distolingual sites (dPD) for first molars (M1s) and M2s of the same quadrant across the follow-up stage (Table 3). As expected, M2s had a greater $\mathrm{dPD}$ than that of M1s was examined when N-M3s was present $(P<0.001)$. Compared to the value at T0, the removal of N-M3s led to a significant reduction in $\mathrm{APD}$ for $\mathrm{M} 2 \mathrm{~s}$ at both the T1 and T2 time points $(P=0.001$ and $P<0.001$, respectively), but no significant change was found in the dPD for M1s $(P>0.05)$. Again, the dPD for M2s 6 months postoperatively (T2) was similar to that of M2s in the M3s $(-)$ group $(P=0.956)$.

Inflammatory biomarkers in GCF

Considering that the concentrations of specific inflammatory biomarkers involved in GCF can reflect the local periodontal immune condition of a tooth, the concentrations of interleukin (IL)-1 $\beta$, matrix metalloproteinase (MMP)-8, and tissue inhibitors of matrix metalloproteinase (TIMP)- 1 were measured in the GCF samples obtained at the baseline (TO) and across the follow-up period ( $\mathrm{T} 1$ and $\mathrm{T} 2$ ) to monitor the immunological changes in the periodontal condition of $\mathrm{M} 2 \mathrm{~s}$ in response to $\mathrm{N}-\mathrm{M} 3$ removal (Fig. 2). Although the removal of N-M3s resulted in no significant changes in IL-1 $\beta$ concentration (Fig. 2a), it was found that, following N-M3 extraction, the concentration of MMP-8 decreased with time (from T0 and T1 to T2), with a significant change in MMP-8 concentration was found at T2 (compared to the value at T0, $P=0.015$, Fig. $2 b$ ). The TIMP- 1 concentration at T2 was also reduced but was not significantly different from the value at T0 (no statistical significance, $P>0.05$, Fig. 2c). Importantly, the MMP-8/TIMP-1 ratio was reduced following $\mathrm{N}-\mathrm{M} 3$ extraction, and it was found to be significantly lower at T1 than that at T0 $(P=0.006$, Fig. $2 \mathrm{~d})$, while the reduction in the MMP-8/TIMP-1 ratio showed no statistical significance at $\mathrm{T} 2$ $(P>0.05)$.
Table 3. Changes in the $\mathrm{dPD}$ of M1s and M2s in participants with neighboring $\mathrm{N}-\mathrm{M} 3 \mathrm{~s}(\mathrm{~N}=26)$ in response to $\mathrm{N}-\mathrm{M} 3$ removal and clinical periodontal indexes of M1s and M2s in participants without neighboring $\mathrm{M} 3 \mathrm{~s}(\mathrm{~N}=28)$

\begin{tabular}{llllll}
\hline Groups & & Teeth & \multicolumn{2}{l}{$\mathrm{dPD} / \mathrm{mm}$ (mean \pm SD) } & \multirow{2}{*}{$P^{\mathrm{b}}$} \\
\cline { 5 - 5 } & & $(\mathrm{M} 2 \mathrm{~s})$ & $\mathrm{M} 1 \mathrm{~s}$ & $\mathrm{M} 2 \mathrm{~s}$ & \\
\hline N-M3s (+) & T0 & 26 & $2.69 \pm 0.47$ & $3.65 \pm 0.85$ & $<0.001$ \\
& $P^{\mathrm{a}}$ & & 0.598 & $<0.001$ & \\
& T1 & 26 & $2.58 \pm 0.52$ & $2.83 \pm 0.51$ & 0.074 \\
& $P^{a}$ & & 0.242 & 0.053 & \\
& $P^{1}$ & & $\mathrm{NS}$ & $<0.001$ & \\
& T2 & 26 & $2.54 \pm 0.37$ & $2.54 \pm 0.51$ & 0.888 \\
& $P^{\mathrm{a}}$ & & 0.043 & 0.956 & \\
& $P^{2}$ & & $\mathrm{NS}$ & $<0.001$ & \\
M3s $(-)$ & & 28 & $2.73 \pm 0.40$ & $2.55 \pm 0.39$ & 0.040 \\
\hline
\end{tabular}

Note: M2s, second molars; N-M3s, nonimpacted third molars; M3s, third molars; N-M3s (+), M2s with neighboring N-M3s; M3s (-), M2s without neighboring $\mathrm{M} 3 \mathrm{~s}$.

$\mathrm{dPD}$, mean probing depth of distobuccal and distolingual sites.

T0, baseline; T1, 3 months after M3 extraction; T2, 6 months after M3 extraction.

$\mathrm{SD}$, standard deviation; $P^{\mathrm{a}}$, compared between N-M3s (-) and N-M3 (+) (T0, T1, or T2); $P^{\mathrm{b}}$, compared within the same period; $P^{1}$, compared between T0 and T1; $P^{2}$, compared between T0 and T2; $P^{1}$ and $P^{2}$ values were adjusted for multiple comparisons by the Bonferroni correction; NS, no significance; because the dPD showed no significant change among T0-T2, pairwise comparisons were not conducted between T0 and T1 (or T0 and T2); the significance value was set at 0.05 .

Microbial composition in subgingival plaque

Considering that the periodontal diseases are initiated by the colonization of subgingival periodontal pathogens, the microbial diversity and composition of the subgingival plaque samples obtained at the baseline (T0) and across the follow-up period ( $\mathrm{T} 1$ and $\mathrm{T} 2$ ) were determined to monitor the microbiological changes in $\mathrm{M} 2$ periodontal condition in response to 

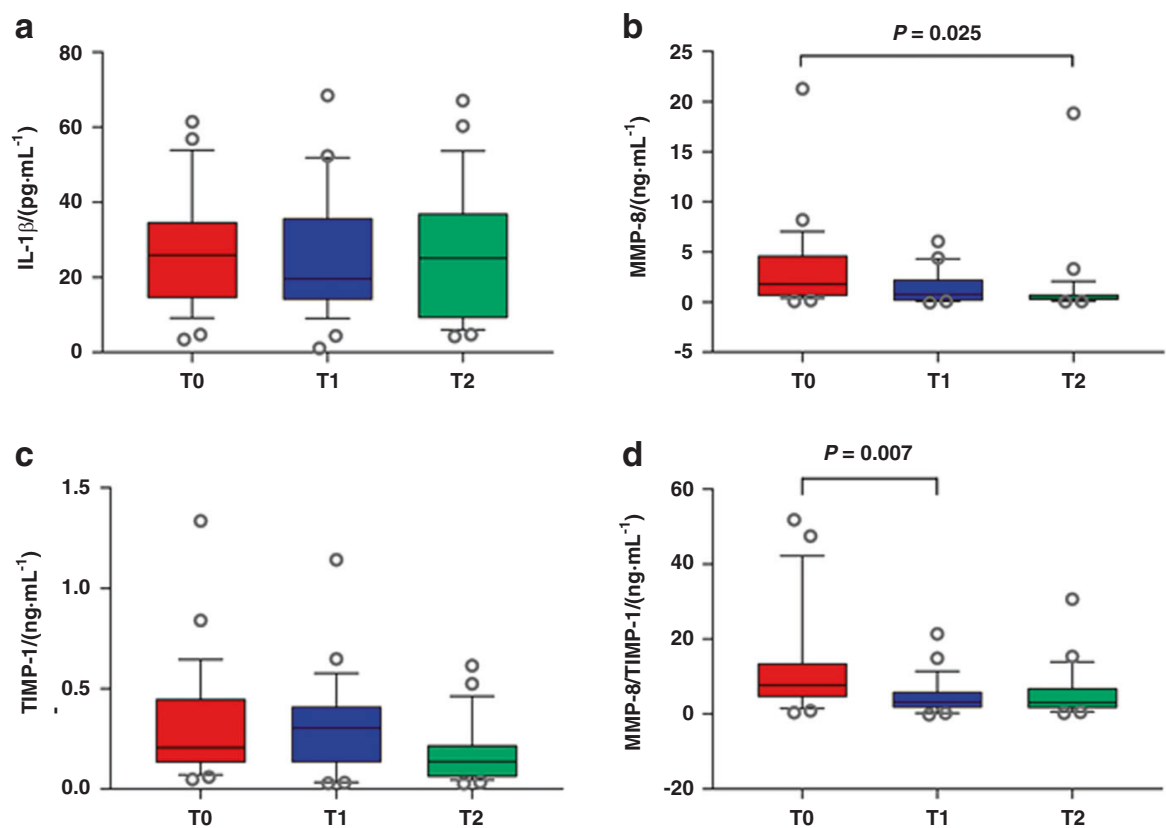

Fig. 2 Changes in inflammatory biomarkers in the GCF of M2s in participants with neighboring N-M3s $(N=26)$ in response to N-M3 removal. Tukey box plots depict the concentrations of a IL-1 $\beta$, b MMP-8, c TIMP-1, and $\mathbf{d}$ the ratio of MMP-8/TIMP-1 involved in GCF. Open circles indicate "outlier" values. $P$, compared between different periods, analyzed by the Friedman test, and were adjusted for multiple comparisons by the Bonferroni correction; T0, before N-M3 removal; T1, 3 months after N-M3 removal; T2, 6 months after N-M3 removal

N-M3 removal (Figs. 3 and 4). Among the 26 participants who underwent tooth extraction, subgingival plaque samples from 8 participants were unqualified; therefore, samples from only 18 participants (obtained at T0-T2) were analyzed using highthroughput sequencing. Finally, a total of 4492092 high-quality sequences (mean per sample: $83167 \pm 7220$; range per sample: 5 069 to 93 753) were generated, and 19194 operational taxonomical units (OTUs) were obtained using a $97 \%$ similarity level.

After the removal of N-M3s, the microbial richness in the subgingival plaque of adjacent M2s increased and then decreased (Fig. 3a, b). Compared with T0, the ACE analysis showed that the microbial richness was significantly reduced at T2 ( $P=0.024$, Fig. 3a). The microbial diversity increased after N-M3 removal (Fig. 3c, d), and compared with T0, a significant increase in the Simpson index was found at T1 and T2 ( $P=0.042$ and $P=0.012$, respectively, Fig. 3d). Moreover, the variations in the microbial community structures were clearly different between $\mathrm{T} 0$ and $\mathrm{T} 1$ (T0 and $\mathrm{T} 2$ ) presented by principal coordinate analysis (PCoA) (Fig. 3e), and significant decreases were found at T1 and T2 $(P<0.001$ and $P<0.001$, respectively, Fig. 3f).

The microbial composition of the subgingival plaque samples of $\mathrm{M} 2 \mathrm{~s}$ in response to N-M3 removal was further analyzed (Fig. 4). At the phylum level, Bacteroidetes, Actinobacteria, Fusobacteria, Proteobacteria, Firmicutes, and Spirochetes were the dominant phyla, accounting for more than $90 \%$ of the sequences (Fig. $4 a$ ). At the genus level, the distributions of the top 35 genera at T0-T2 were different in the heatmap (Fig. 4b). In addition, pathogenic genera such as unidentified Prevotellaceae and Streptococcus were significantly decreased at T2 compared to T0 $(P<0.001$ and $P=$ 0.009 , respectively, Fig. $4 c$, d). Compared to T0, the relative abundance of Actinobacteria was decreased at T1 and T2 $(P=$ 0.006 and $P>0.05$, Fig. 4e), while Fusobacteria, Fusobacterium, and Campylobacter were significantly increased at T2 $(P<0.001, P=$ 0.003 , and $P=0.006$, respectively, Fig. $4 \mathrm{f}-\mathrm{h}$ ).

\section{DISCUSSION}

In recent years, the negative impact of $\mathrm{N}-\mathrm{M} 3 \mathrm{~s}$, as a common clinical problem (Supplementary Fig. 1), has attracted increasing attention from dentists. ${ }^{5,6}$ However, there is limited evidence from the laboratory-based assessments of the periodontal risks associated with N-M3s. We also conducted a pairwise comparison of the periodontal condition of bilaterally matched quadrants of $\mathrm{M} 2 \mathrm{~s}$ with neighboring N-M3s or without M3s from the enrolled participants and found that the presence of $\mathrm{N}-\mathrm{M} 3 \mathrm{~s}$ was associated with increased PD and dPD of neighboring M2s and an increased abundance of pathogenic microbiomes within subgingival plaque compared to the absence of M3s (data not shown). Thus, in this 6-month longitudinal study, we investigated the clinical, immunological, and microbiological changes in the periodontal condition around $\mathrm{M} 2 \mathrm{~s}$ following removal of neighboring N-M3s. We found that N-M3 removal was associated with an improvement of periodontal condition around neighboring $\mathrm{M} 2 \mathrm{~s}$, including superior clinical indexes, decreased GCF inflammatory biomarkers, and reduced pathogenic microbiome distribution within the subgingival plaque.

Regarding clinical periodontal indexes, our data support the findings of previous studies, showing beneficial changes in variables such as PD and $d P D$ for $M 2 s$ in response to neighboring $M 3$ removal. ${ }^{10,13,14}$ However, the reduction in PLI and the prevalence of PD5+ in the present study did not reach statistical significance (Table 2), which may be due to the strict inclusion/exclusion criteria, and the fact that most of the M3s in our study were located in maxillary (87\%) while previous work focused on mandibular M3s. All subjects who came to our school for oral hygiene maintenance purposes during the investigation period were screened according to inclusion/exclusion criteria and their consent to have the N-M3s removed (Fig. 1). The higher proportion of targeted quadrants located in the maxillary supported our previous cross-sectional studies of N-M3s. ${ }^{6,8,11}$ Both radiological and clinical investigations showed that there were more N-M3s in the maxilla, while I-M3s were more likely to be in the mandible. ${ }^{5,9}$ The different influences of $\mathrm{N}-\mathrm{M} 3 \mathrm{~s}$ in maxilla and mandible have been discussed previously. We found that, compared to maxillary N-M3s, N-M3s located in the mandible showed greater risks of periodontal pathology in neighboring $M 2 s . s^{6,8,9,11}$ Separate analysis of the targeted maxillary and mandibular quadrants would be optimal, however, since there 


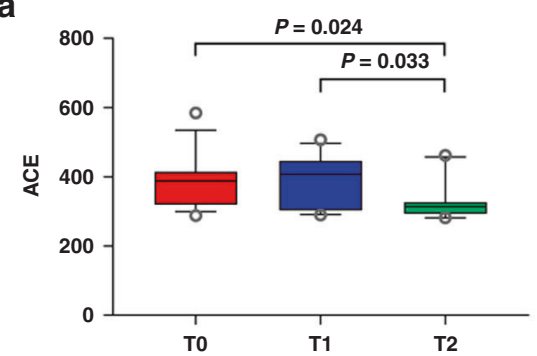

C

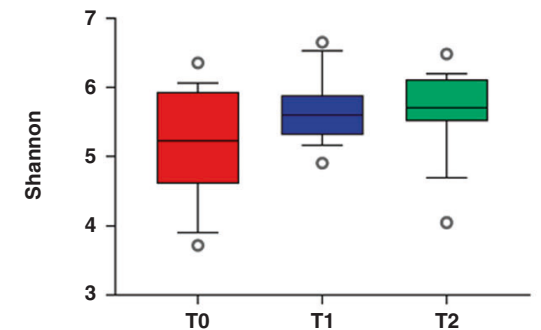

e

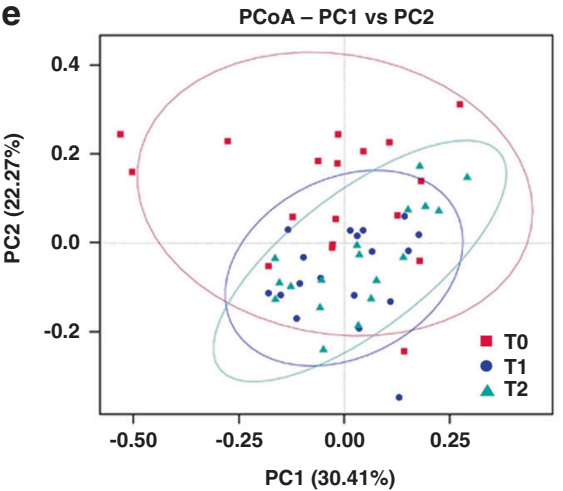

b

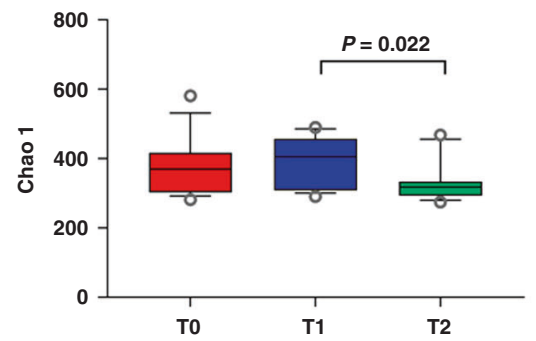

d

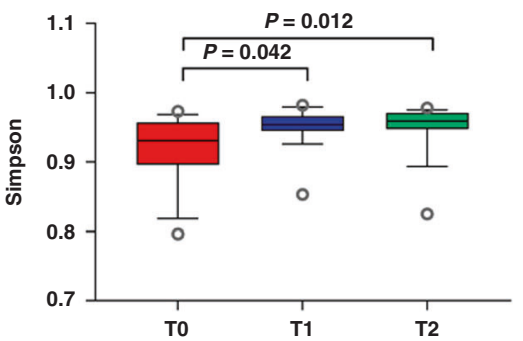

f
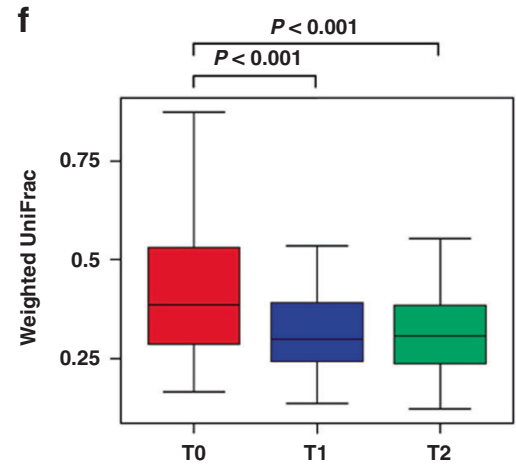

Fig. 3 Changes in the microbial diversity of the subgingival plaque of M2s in participants with neighboring $N-M 3 s$ ( $N=18$ ) in response to N-M3 removal. Community richness was evaluated by a ACE and b Chao 1, while diversity was analyzed by $\mathbf{c}$ Shannon and $\mathbf{d}$ Simpson indexes. e Principal coordinate analysis (PCoA) plot based on weighted UniFrac distances and $\mathbf{f}$ weighted UniFrac distances depict beta diversity. T0, before N-M3 removal; T1, 3 months after N-M3 removal; T2, 6 months after N-M3 removal

was only one mandibular targeted quadrant that underwent N-M3 extraction, this analysis was not conducted. In the present study, the degree of clinical improvement of PD and dPD for M2s was further compared with control subjects lacking M3 (Tables 2 and 3). The reductions in $\mathrm{PD}$ and $\mathrm{dPD}$ after $\mathrm{N}-\mathrm{M} 3$ removal reached a level similar to that of the control subjects. Interestingly, no significant changes in PD5+ prevalence or GCF volume were found postoperatively, while BOP+ prevalence and GCF volume were greater than those of the control. These results may partly explain the relatively low percentage of periodontal healing of M2s with deep PD (PD $\geq 5 \mathrm{~mm}$ ) observed by Sun et al..$^{11}$ In this region around M3s, where accumulated microbial biofilms are difficult to be completely eradicated by mechanical methods, ${ }^{15}$ periodontal pathogens colonize readily and are capable of stimulating inflammatory and immune activities. ${ }^{16-18}$ As a result, patients are often at risk for periodontal diseases in the M3 region, and the presence of M3s may cause periodontal damage to their neighboring M2s.

In addition to the analysis of GCF volume, GCF is widely used to investigate the periodontal inflammatory biomarkers because of its close proximity to the periodontium. Studies have identified multiple biomarkers in the GCF during the inflammatory process of periodontal diseases and treatments. ${ }^{19,20}$ Among those biomarkers, IL-1 $\beta$ and MMP-8 have shown diagnostic potential for periodontitis. ${ }^{21-23}$
IL-1 $\beta$ participates in alveolar bone resorption and is expected to be significantly downregulated after periodontal treatments. ${ }^{19,24}$ It was demonstrated that an increased concentration of IL-1 $\beta$ was present in GCF if PD5+ was detected around M3s compared with patients with PD less than $5 \mathrm{~mm} .{ }^{18}$ In the current investigation, we did not detect significant differences in IL-1 $\beta$ concentration (Fig. 2a). This could be explained by the lack of a significant reduction in the prevalence of PD5 + in M2s in response to N-M3 removal.

MMP-8 (or collagenase 2), as a prominent collagenase, is involved in the degradation of the extracellular matrix and basement membrane. ${ }^{25-27}$ MMPs are controlled and inhibited by specific tissue inhibitors, mainly TIMP-1, and a shift in the balance between MMP-8 and TIMP-1 can lead to the progression of periodontal diseases. ${ }^{28,29}$ In this study, our results showed that the MMP-8 concentration and the MMP-8/TIMP-1 ratio in GCF decreased following N-M3 extraction: MMP-8 concentration was significantly reduced at $T 2$, and the MMP-8/TIMP-1 ratio was significantly reduced at $\mathrm{T} 1$ (Fig. $2 \mathrm{~b}, \mathrm{~d}$ ). As no other studies have explored concentrations of MMP-8 and TIMP-1 around M2s, the results of the present study cannot be compared with those in the literature. However, these reductions were associated with tissue reattachment in the healing process and were in agreement with earlier studies showing that clinical indexes improved with decreased concentrations of MMP-8 after 
a

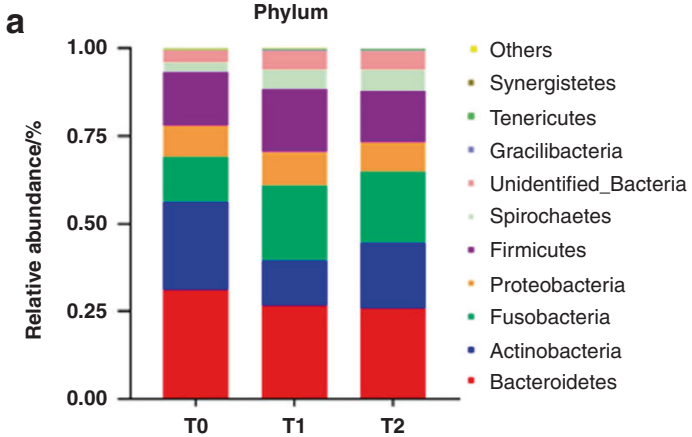

b

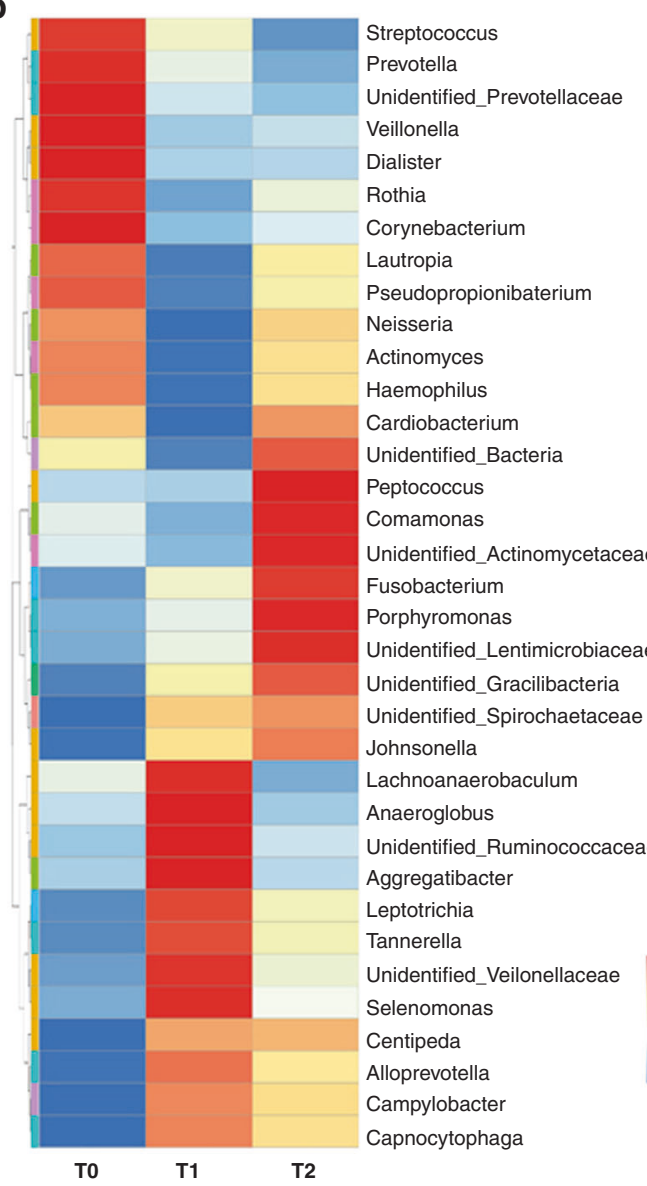

C

unidentified Prevotellaceae *

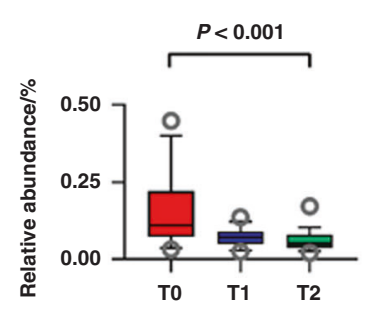

e

Actinobacteria

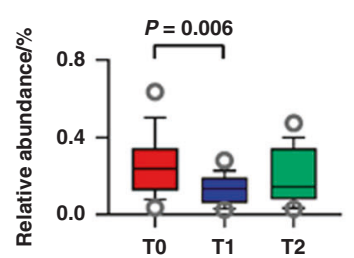

g

Fusobacterium

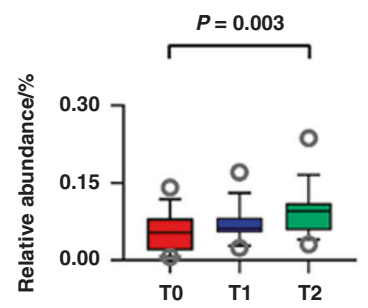

d

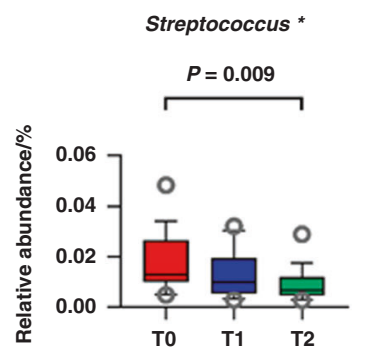

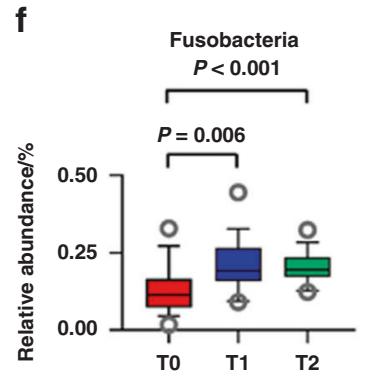

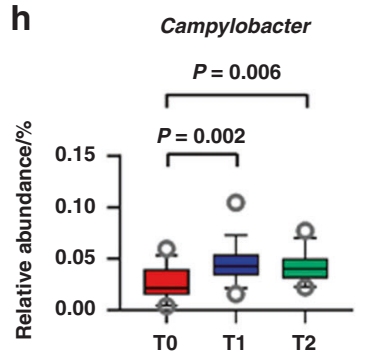

Fig. 4 Changes in the microbial composition of subgingival plaque of $\mathrm{M} 2 \mathrm{~s}$ in participants with neighboring $\mathrm{N}-\mathrm{M} 3 \mathrm{~s}(\mathrm{~N}=18)$ in response to $\mathrm{N}-\mathrm{M} 3$ removal. a Top ten relative abundance of bacterial phyla distributed within the subgingival plaque. b Heatmap shows the relative abundance of the top 35 genera distributed within the subgingival plaque. Colors reflect the abundance of genera from high (red) to low (blue). c, $\mathbf{d}$ The changes in the genera unidentified Prevotellaceae and Streptococcus, presented by Tukey box plots. e-h The changes in the phyla Actinobacteria and Fusobacteria as well as genera Fusobacterium and Campylobacter, presented by Tukey box plots. T0, before N-M3 removal; T1, 3 months after N-M3 removal; T2, 6 months after N-M3 removal; *, putative periodontopathic genera

periodontal treatments. ${ }^{30,31}$ The concentration of TIMP-1 has been reported in the literature to increase or decrease after various treatments, ${ }^{32,33}$ indicating that the regulation of TIMP-1 may not be solely dependent on MMPs. The observations of the MMP-8/TIMP-1 ratio from our study were consistent with previous investigations. A higher MMP-8/TIMP-1 ratio was detected in patients with periodontal inflammation, ${ }^{34,35}$ and it was positively associated with the periodontal inflammatory burden index. ${ }^{36}$ In a paper by Teixeira et al. ${ }^{37}$, MMP-8 concentration and MMP-8/TIMP-1 ratio were significantly higher in patients with $P D \geq 6 \mathrm{~mm}$. In addition to local inflammatory status, several studies suggested that M3s were associated with higher systemic inflammation than controls, ${ }^{38}$ and oxidative stress biomarkers were reduced significantly after $M 3$ extraction. ${ }^{39}$

The microbiological findings showed that $M 3$ extraction effectively reduced microbial richness and increased microbial diversity following N-M3 removal. The relatively high diversity, species homogeneity, and sample-to-sample similarity observed in T2 implied a more complex community after extraction, thus being healthier and more stable (Fig. 3). ${ }^{40,41}$ In other words, before $\mathrm{N}-\mathrm{M} 3$ extraction, there were more microbes with low relative 
abundance at baseline. Moreover, the results of PCoA revealed that the microbial community structures significantly changed after M3 extraction, showing greater concentration in samples at T2 compared to baseline. The relationship among the microbial communities in different samples based on the unweighted pair group method with arithmetic mean (UPGMA) clustering analysis also suggested that samples at baseline could be easily discriminated from the samples at T1 and T2 (Supplementary Fig. 2). This suggested that the microbial community might have undergone a similar healing process postoperatively. These data are in accordance with Shen et al. who observed greater similarity of microbial composition in patients without adverse reactions following $M 3$ extraction, compared with patients diagnosed with dry socket. ${ }^{42}$ Our data were also in accordance with previous investigators who found remaining Shannon diversity values at pretreatment levels up to 6 months after extraction. ${ }^{43}$ However, the increased Simpson index after M3 extraction could be due to the continuous outflow of GCF from the gingival sulcus, which could protect the subgingival microenvironment. In addition, the subgingival microbiome is site-specific and is mainly influenced by the periodontal depth and local inflammation. ${ }^{44}$

In the current study, the relative abundance of Fusobacteria was significantly altered among time points. Fusobacteria belongs to late colonizers of the healthy core oral microbiome, ${ }^{45,46}$ and they significantly increased in response to N-M3 removal. At the genus level, unidentified Prevotellaceae and Streptococcus significantly decreased 6 months postoperatively. Some Prevotella and Streptococcus species have been identified as putative periodontal pathogens and are closely related to the orange complex. ${ }^{47-50}$ Our findings indicated that unidentified Prevotellaceae could be associated with the onset or persistence of periodontal inflammation involved in the M3 region. The genus Streptococcus has been previously reported to have a significantly higher abundance of in response to nonsurgical periodontal therapy. ${ }^{51,52}$ In contrast, our findings showed a decreased abundance of Streptococcus. A possible explanation could be the relatively longer follow-up time in our study and the differences in participants. In addition, Fusobacterium and Campylobacter significantly increased at T2, and both of them have been detected in the healthy oral cavity. ${ }^{45}$ The correlations between the periodontopathic microbiome and inflammatory biomarkers were further analyzed by Spearman correlation (Supplementary Table). Although no statistical significance was observed, the relative abundance of unidentified Prevotellaceae was positively correlated with inflammatory biomarkers in GCF.

Interestingly, our findings in comparisons of bilaterally matched quadrants also showed significantly higher abundance of Fusobacteria and lower abundance of unidentified Prevotellaceae in the M3s (-) group (data will be published elsewhere), which was similar to patients who received N-M3 removal at the 6-month follow-up. These results indicated that the relationship between Fusobacteria and unidentified Prevotellaceae could have crucial roles in the negative influence of $\mathrm{N}-\mathrm{M} 3 \mathrm{~s}$. Previous studies also demonstrated a greater proportion of putative periodontal pathogens in the M3 region than incisor sites, ${ }^{16}$ and these pathogens may initiate periodontitis in young adults. ${ }^{18}$ In that case, protective $M 3$ extraction would be beneficial since the extraction made it accessible for the distal surface of $M 2 s$ to maintain better self-cleaning, forming a healthy periodontium, and may help prevent the development and progression of periodontitis.

We are well aware of the strengths and limitations of our study. On the one hand, this study combines the clinical, immunological, and microbiological parameters, providing comprehensive evidence on which to base future research into the impact of N-M3s on the periodontal health of neighboring teeth. On the other hand, the nonrandomized nature of the study design and the relatively short follow-up time are limitations of the study. The removal of M3s carries costs and risks, therefore, conducting a well-designed randomized (or split mouth) clinical trial is challenging. However, randomized methods were applied to select study quadrants. In addition, there were no significant differences in clinical periodontal indexes or immunological findings between 3 and 6 months postoperatively, which indicated that the periodontium of $\mathrm{M} 2 \mathrm{~s}$ was in a stable state 6 months after the extraction.

Over time, our group has conducted several studies on the negative periodontal influence of N-M3s. First, we noticed the risk of alveolar bone resorption of neighboring $\mathrm{M} 2 \mathrm{~s}$ from the radiological investigation. ${ }^{6}$ Then, we discovered clinical manifestations of deeper PD with the presence of adjacent N-M3s. ${ }^{8,9}$ In search of a clinical strategy for governing N-M3s, we demonstrated that $\mathrm{M} 2 \mathrm{~s}$ exhibited improved periodontal conditions following $\mathrm{N}$ M3 removal. ${ }^{11}$ This study explored the immunological and microbiological reasons for the improvement of the periodontal condition around adjacent teeth after N-M3 extraction. However, it is still too early to make a conclusion based on current studies. We are currently exploring the clinical strategy and personalized treatment plan for $\mathrm{N}-\mathrm{M} 3 \mathrm{~s}$ through population-based multicenter clinical trials. In the future, we plan to further explore the alterations of a larger array of biomarkers in other body fluids (such as blood and saliva) and verify the periodontal hazards of the pathogenic microbiome found in this article through animal experiments. Future studies on the current topic are therefore recommended, and the above limitations should be taken into consideration.

\section{MATERIALS AND METHODS}

Ethics of the study

The present cohort study fully complied with the Declaration of Helsinki on experimentation involving humans. Verbal and written information describing the nature of the study was given to all enrolled participants and signed informed consent forms were obtained prior to enrollment of the subjects in the investigation. The Ethics Committee and Institutional Review Board of School of Stomatology at the Fourth Military Medical University (FMMU) approved the protocol (ethics committee protocol number: 2017034; date of registration: 22 December 2017).

\section{Inclusion and exclusion criteria}

This study was conducted at the School of Stomatology FMMU (Xi'an, China). Consecutive subjects with at least one quadrant having an intact $\mathrm{M} 1, \mathrm{M} 2$, and $\mathrm{N}-\mathrm{M} 3$ were screened, and those who decided to undergo $\mathrm{N}-\mathrm{M} 3$ extraction were asked to participate in this investigation. N-M3 was defined as an asymptomatic third molar that reached the occlusal plane, lacked rotation, and could be examined on all five surfaces, regardless of its functionality. ${ }^{8}$ The recruited subjects were assigned to the N-M3s (+) group. At the same time, subjects with at least one quadrant having intact $\mathrm{M} 1$ and $\mathrm{M} 2$ but no M3 (congenitally absent or extracted more than 6 months prior according to case history and radiography) were also recruited for the control for clinical assessment as the M3s (-) group. The subject-related inclusion criteria were as follows: (i) age of $\geq 18$ years of age; (ii) no crown or bridge prostheses, severe caries, or advanced periodontitis (PD $\geq 7 \mathrm{~mm}$ and/or radiographic imaging of bone resorption more than $20 \%$ of the tooth length) in the examined quadrant ${ }^{38}$; and (iii) had at least 20 natural permanent teeth, with functional occlusal contact in dentistry.

Subjects were excluded if they (i) were smokers; (ii) were females who were pregnant or lactating; (iii) had any systemic condition that could influence the course of periodontal disease (e.g., diabetes, hypertension, or a history of radiation or cancer therapy); (iv) had received orthodontic treatment; (v) had undergone periodontal treatment within 6 months, received antibiotics/ anti-inflammatory drugs/oral contraceptives within 3 months, 
used mouthwash that contained antibiotics within 3 months, or (vi) suffered acute dental infections in need of emergency treatment.

Sample size estimation

The primary outcome of the present study was the PD change of $\mathrm{M} 2 \mathrm{~s}$ in response to neighboring N-M3 removal; hence, similar to a previous study, ${ }^{11}$ subject size calculation was performed according to the PD change using specialized software ( $G^{*}$ Power version 3.0.8, Heinrich Heine University, Düsseldorf, Germany). The power calculation analysis revealed that to detect a $0.5 \mathrm{~mm}$ difference in PD at the 0.05 probability level with a power of $80 \%$, the required minimum sample size for $\mathrm{N}-\mathrm{M} 3$ extraction was 21 . Assuming a $20 \%$ drop-out rate, a minimum of 24 subjects was suggested to be recruited.

\section{Recruitment of subjects}

All subjects who came to our school for oral hygiene maintenance purposes during the investigation period were screened as potential participants, and those recruited to this study were based on their agreement to participate and met the inclusion/ exclusion criteria. For N-M3s (+) subjects, their recruitment into the investigation was also dependent on their agreement to undergo tooth extraction at a certain stage of the study.

\section{Study design}

In this 6-month longitudinal study, changes in clinical periodontal indexes, inflammatory biomarkers in GCF, and the composition of subgingival plaque of $\mathrm{M} 2 \mathrm{~s}$ in response to $\mathrm{N}-\mathrm{M} 3$ removal were analyzed. For the $\mathrm{N}-\mathrm{M} 3 \mathrm{~s}(+)$ group, clinical periodontal indexes and GCF and plaque samples were collected prior to the surgical procedure (baseline, T0) as well as at the follow-up stage (3 months postoperatively, T1; 6 months postoperatively, T2). Since the subject-specific microbiota was distributed in periodontally healthy individuals, ${ }^{53}$ immunological and microbial analyses were only performed on subjects who undergone N-M3 removal. Therefore, for the M3s (-) group, only clinical periodontal indexes were collected at baseline once the subjects were successfully enrolled in the study to determine the degree of clinical improvement.

The decision to extract $\mathrm{N}-\mathrm{M} 3 \mathrm{~s}$ was made by experienced dentists and they were not involved in any research procedure of the present study. Besides observing the clinical, immunological, and microbiological changes before M3 extraction and postoperatively, the researchers of this study did not interfere with the decision to extract teeth. The N-M3s removal surgery was performed under local anesthesia (Perimacaine, Z.I. du Phare, Merignac, France). For all patients, soft tissue flaps, tooth section, and/or osteotomy were unnecessary, and once the tooth was removed, the socket was carefully inspected. Afterward, patients were instructed to take local disinfectant buccal tablets ( $2 \mathrm{mg}$; Cetylpyridinium Chloride Buccal Tablets, HWANG'S Pharmaceutical Co., Ltd., Taiwan) every $6 \mathrm{~h}$ for 3 days. Analgesics (60 mg; Loxoprofen Sodium Capsules, QIDU Pharmaceutical Co. Ltd., Shandong, China) were prescribed every $8 \mathrm{~h}$ for 3 days when necessary. ${ }^{11}$ Participants were automatically excluded if they (i) did not complete the follow-up, (ii) received antibiotics/anti-inflammatory drugs, or (iii) underwent periodontal, restorative, or orthodontic treatment during the follow-up period. If a participant had more than one eligible quadrant for investigation, only one quadrant was randomly selected for research.

Sociodemographic information of the participants

All participants were asked to answer all questions on a questionnaire including age, gender, ethnicity, education level, dental scaling, and dental visit frequency. However, only those who completed the study were used for analysis.
Clinical periodontal indexes

For all participants, an experienced periodontist recorded the PD, $\mathrm{BOP}$, and PLI of the molars located in the examined quadrant; PD and BOP were examined at six sites (mesiobuccal, midbuccal, distobuccal, mesiolingual, midlingual, and distolingual) using a manual periodontal probe (Hu-Friedy, Chicago, IL, USA). ${ }^{11}$

PD was recorded as the mean value of the six sites. PD5+ was recorded as at least one probing site $\geq 5 \mathrm{~mm}$. BOP + was recorded when at least three sites of M2 showed any trace of blood after probing. The PLI of the buccal and lingual surfaces was recorded according to the scoring criteria previously described in the literature. ${ }^{11}$ Specifically, dPD was recorded as the mean PD of distobuccal and distolingual sites.

GCF sampling and inflammatory biomarkers

For participants receiving tooth extraction, GCF samples were collected at T0-T2 from the distobuccal and distolingual sites of the M2s of the targeted quadrant by means of sterile PerioPaper strips (Oraflow, Smithtown, NY, USA), and performed after airdrying, isolation with cotton rolls, and removal of supragingival plaque. The paper strips were introduced into the sulcus until resistance was felt and kept in situ for $30 \mathrm{~s}$. Strips were discarded when contaminated with visible blood or saliva. Subsequently, each strip was measured for fluid volume with a precalibrated Periotron 8000 (Oraflow Inc.). Then, two strips were pooled and eluted into an Eppendorf tube containing $500 \mu \mathrm{L}$ of phosphatebuffered saline (PBS) and placed immediately into dry ice. The readings from the Periotron 8000 were converted to actual volume $(\mu \mathrm{L})$ according to the manufacturer's instructions. Each sample was vortexed and centrifuged at $3000 \times g$ for $10 \mathrm{~min}$. Then, the paper strips were removed, and the supernatant was stored at $-80^{\circ} \mathrm{C}$ until the day of the analysis. ${ }^{54}$ Concentrations of IL-1 $\beta$, MMP-8, and TIMP-1 involved in the GCF samples were determined using commercially available enzyme-linked immunosorbent assay kits from Quantikine (R\&D Systems, Inc., MN, USA) according to the manufacturer's instructions. The detection levels of the kits were $1 \mathrm{pg} / \mathrm{mL}$ for IL-1 $\beta$, assay range $3.9-250 \mathrm{pg} \cdot \mathrm{mL}^{-1}$, $0.06 \mathrm{ng} \cdot \mathrm{mL}^{-1}$ for MMP-8, assay range $0.2-10 \mathrm{ng} \cdot \mathrm{mL}^{-1}$, and $0.08 \mathrm{ng} \cdot \mathrm{mL}^{-1}$ for TIMP-1, assay range $0.2-10 \mathrm{ng} \cdot \mathrm{mL}^{-1}$. All samples were assayed in duplicate. The concentrations of IL-1 $\beta$, MMP-8, and TIMP-1 in each sample were then corrected based on the volume of GCF.

Subgingival plaque sampling and microbial composition analysis In parallel to and after GCF collection, subgingival plaque samples were collected using a sterile Gracey curette from the sulcus of the distal site of targeted $\mathrm{M} 2 \mathrm{~s}$, placed into an Eppendorf tube containing $1000 \mu \mathrm{L}$ of PBS, and immediately stored at $-80^{\circ} \mathrm{C}$ for DNA extraction. The microbial DNA was isolated as previously described. ${ }^{44}$ In brief, genomic DNA was isolated and purified from the subgingival plaque samples using a commercially available kit (QIAamp DNA Mini Kit, QIAGEN Sciences, USA) according to the manufacturer's instructions. The V3-V4 hypervariable regions of the $16 \mathrm{~S}$ rRNA gene were amplified using the 515F/608R universal primer with the barcode and subjected to high-throughput sequencing using the Illumina NovaSeq6000 platform. The raw sequencing data were processed using the pipeline tools of QIIME 1.9.1 (http://qiime.org/scripts/split_libraries_fastq.html). After trimming and filtering, high-quality sequences were aligned to the Silva 132 database (http://www.arb-sliva.de/) and clustered into OTUs using Uparse software (Uparse v7.0.1001, http://drive5.com/ uparse/) at a $97 \%$ similarity level.

Statistical analysis

Data were recorded and analyzed at the tooth level. Missing values due to loss to follow-up were deleted. Decisions about whether to use parametric or nonparametric tests were made based on the results of the Kolmogorov-Smirnow test for normal 
distribution. Nonparametric tests were used for intra- and intergroup comparisons (Wilcoxon signed-rank test for matched comparisons, Mann-Whitney $U$ test for intergroup comparisons, and Friedman test for comparisons among multiple periods). The chi-square test and Cochran's $Q$ test were used to compare the proportions of PD5 + and $\mathrm{BOP}+$. The diversity of the microbial community was determined by alpha diversity indices, including the ACE, Chao1, Shannon, and Simpson indices. The comparison of the microbial structure was conducted using PCOA based on weighted UniFrac distance. The distribution of the relative abundance of the top 35 genera was plotted using a heatmap. MetaStats analysis was used to determine the statistical significance of differences in relative abundance at different time points. The two-sided significance value was set at 0.05 . For multiple testing, the $P$ value was further adjusted by the Bonferroni correction. All statistical analyses were performed with QIIME 1.9.1, R 2.15.3 (R Foundation, Vienna, Austria), and SPSS (v20.0, IBM, Chicago, IL, USA).

\section{CONCLUSION}

In this longitudinal study, we investigated how the periodontal condition changed in response to neighboring N-M3 removal across a 6-month period. For the first time, the present study demonstrated that $\mathrm{N}-\mathrm{M} 3$ removal was associated with an improvement of the periodontal condition around neighboring teeth, including superior clinical parameters, decreased GCF inflammatory biomarkers, and reduced pathogenic microbiome distribution within subgingival plaque. Our findings provide direct evidence that N-M3s exert adverse effects on the periodontal health of adjacent M2s. Although prophylactic removal of N-M3s remains controversial, we suggest that an early medical decision should be made on N-M3s before the neighboring teeth are irretrievably damaged.

\section{DATA AVAILABILITY}

The raw sequence data are available in the NCBI Sequence Read Archive under BioProject PRJNA656079, and data that support the other findings in this study are available from the corresponding author upon reasonable request.

\section{ACKNOWLEDGEMENTS}

This study was financially supported by the Major Research Program of the National Natural Science Foundation of China (Beijing, Subproject No. 81991503) and the Changjiang Scholars Program of the Ministry of Education of the People's Republic of China (2016).

\section{AUTHOR CONTRIBUTIONS}

Y.T. and L.J.S. conducted the investigation, collected data, and drafted the paper. H.L.Q. and Y.Y. participated in the conceptualization, methodology, and visualization of the study. F.M.C. was the main supervisor and initiator of this study. All authors reviewed, revised, and finalized the paper.

\section{ADDITIONAL INFORMATION}

The online version of this article (https://doi.org/10.1038/s41368-020-00108-y) contains supplementary material, which is available to authorized users.

Competing interests: The authors declare no competing interests.

Ethical approval: This study was approved by the Ethics Committee and Institutional Review Board, School of Stomatology, FMMU (date of approval: December 22, 2017; approval number: IRB-REV-2017034).

\section{REFERENCES}

1. Haug, R. H., Abdul-Majid, J., Blakey, G. H. \& White, R. P. Evidenced-based decision making: the third molar. Dent. Clin. North Am. 53, 77-96, ix (2009).
2. Steed, M. B. The indications for third-molar extractions. J. Am. Dent. Assoc. 145, 570-573 (2014)

3. Nunn, M. E. et al. Retained asymptomatic third molars and risk for second molar pathology. J. Dent. Res. 92, 1095-1099 (2013).

4. Venta, I., Vehkalahti, M. M. \& Suominen, A. L. What kind of third molars are disease-free in a population aged 30 to 93 years? Clin. Oral. Investig. 23, 1015-1022 (2019).

5. Kindler, S. et al. Third molars and periodontal damage of second molars in the general population. J. Clin. Periodontol. 45, 1365-1374 (2018).

6. Li, Z. B., Qu, H. L., Zhou, L. N., Tian, B. M. \& Chen, F. M. Influence of non-impacted third molars on pathologies of adjacent second molars: a retrospective study. $J$. Periodontol. 88, 450-456 (2017).

7. Chou, Y. H., Ho, P. S., Ho, K. Y., Wang, W. C. \& Hu, K. F. Association between the eruption of the third molar and caries and periodontitis distal to the second molars in elderly patients. Kaohsiung J. Med. Sci. 33, 246-251 (2017).

8. Li, Z. B. et al. Nonimpacted third molars affect the periodontal status of adjacent teeth: a cross-sectional study. J. Oral. Maxillofac. Surg. 75, 1344-1350 (2017).

9. Qu, H. L. et al. Effect of asymptomatic visible third molars on periodontal health of adjacent second molars: a cross-sectional study. J. Oral. Maxillofac. Surg. 75, 2048-2057 (2017).

10. Passarelli, P. C. et al. Influence of mandibular third molar surgical extraction on the periodontal status of adjacent second molars. J. Periodontol. 90, 847-855 (2019).

11. Sun, L. J. et al. Impacts of non-impacted third molar removal on the periodontal condition of adjacent second molars. Oral. Dis. 26, 1010-1019 (2020).

12. Kinane, D. F., Stathopoulou, P. G. \& Papapanou, P. N. Periodontal diseases. Nat. Rev. Dis. Prim. 3, 17038 (2017).

13. Petsos, H., Korte, J., Eickholz, P., Hoffmann, T. \& Borchard, R. Surgical removal of third molars and periodontal tissues of adjacent second molars. J. Clin. Periodontol. 43, 453-460 (2016).

14. Pham, T. A. V. \& Nguyen, N. H. Periodontal status of the adjacent second molar after impacted mandibular third molar surgical extraction. Contemp. Clin. Dent. 10, 311-318 (2019).

15. Fisher, E. L., Blakey, G. H., Offenbacher, S., Phillips, C. \& White, R. P. Jr Mechanical debridement of subgingival biofilm in participants with asymptomatic third molars does not reduce deeper probing depths in the molar regions of the mouth. J. Oral. Maxillofac. Surg. 71, 467-474 (2013).

16. Mansfield, J. M., Campbell, J. H., Bhandari, A. R., Jesionowski, A. M. \& Vickerman, M. M. Molecular analysis of $16 \mathrm{~S}$ rRNA genes identifies potentially periodontal pathogenic bacteria and archaea in the plaque of partially erupted third molars. $J$. Oral. Maxillofac. Surg. 70, 1507-1514 e1501-1506 (2012).

17. White, R. P. Jr et al. Microbial complexes detected in the second/third molar region in patients with asymptomatic third molars. J. Oral. Maxillofac. Surg. 60, 1234-1240 (2002).

18. White, R. P. Jr et al. Inflammatory mediators and periodontitis in patients with asymptomatic third molars. J. Oral. Maxillofac. Surg. 60, 1241-1245 (2002).

19. Stadler, A. F. et al. Gingival crevicular fluid levels of cytokines/chemokines in chronic periodontitis: a meta-analysis. J. Clin. Periodontol. 43, 727-745 (2016).

20. Tsuchida, S., Satoh, M., Takiwaki, M. \& Nomura, F. Current status of proteomic technologies for discovering and identifying gingival crevicular fluid biomarkers for periodontal disease. Int. J. Mol. Sci. 20, 86 (2018).

21. Alassy, H., Parachuru, P. \& Wolff, L. Peri-implantitis diagnosis and prognosis using biomarkers in peri-implant crevicular fluid: a narrative review. Diagnostics. 9, 214 (2019).

22. Almehmadi, A. H. \& Alghamdi, F. Biomarkers of alveolar bone resorption in gingival crevicular fluid: a systematic review. Arch. Oral. Biol. 93, 12-21 (2018).

23. Nazar Majeed, Z., Philip, K., Alabsi, A. M., Pushparajan, S. \& Swaminathan, D. Identification of gingival crevicular fluid sampling, analytical methods, and oral biomarkers for the diagnosis and monitoring of periodontal diseases: a systematic review. Dis. Markers 2016, 1804727 (2016).

24. Cheng, R., Wu, Z., Li, M., Shao, M. \& Hu, T. Interleukin-1beta is a potential therapeutic target for periodontitis: a narrative review. Int. J. Oral. Sci. 12, 2 (2020).

25. Costa, F. O. et al. Cytokine levels in crevicular fluid associated with compliance during periodontal maintenance therapy. Clin. Oral. Investig. 23, 3517-3526 (2019).

26. Konopka, L., Pietrzak, A. \& Brzezinska-Blaszczyk, E. Effect of scaling and root planing on interleukin-1 beta, interleukin- 8 and MMP-8 levels in gingival crevicular fluid from chronic periodontitis patients. J. Periodontal Res. 47, 681-688 (2012).

27. Sorsa, T. et al. Analysis of matrix metalloproteinases, especially MMP-8, in gingival creviclular fluid, mouthrinse and saliva for monitoring periodontal diseases. Periodontology 70, 142-163 (2016).

28. Boelen, G. J. et al. Matrix metalloproteinases and inhibitors in dentistry. Clin. Oral. Investig. 23, 2823-2835 (2019). 
29. Rathnayake, N. et al. Salivary biomarkers of oral health: a cross-sectional study. J. Clin. Periodontol. 40, 140-147 (2013).

30. Chen, Y. W., Hsieh, O., Chen, Y. A., Chiou, L. L. \& Chang, P. C. Randomized controlled clinical effectiveness of adjunct $660-\mathrm{nm}$ light-emitting diode irradiation during non-surgical periodontal therapy. J. Formos. Med. Assoc. 119, 157-163 (2020).

31. Chiang, C. P., Hsieh, O., Tai, W. C., Chen, Y. J. \& Chang, P. C. Clinical outcomes of adjunctive indocyanine green-diode lasers therapy for treating refractory periodontitis: a randomized controlled trial with in vitro assessment. J. Formos. Med. Assoc. 119, 652-659 (2020).

32. Emingil, G. et al. Matrix metalloproteinase (MMP)-8 and tissue inhibitor of MMP-1 (TIMP-1) gene polymorphisms in generalized aggressive periodontitis: gingival crevicular fluid MMP-8 and TIMP-1 levels and outcome of periodontal therapy. J. Periodontol. 85, 1070-1080 (2014).

33. Turkoglu, $O$. et al. The effect of adjunctive chlorhexidine mouthrinse on GCF MMP-8 and TIMP-1 levels in gingivitis: a randomized placebo-controlled study. BMC Oral. Health 14, 55 (2014).

34. Ince, G. et al. Clinical and biochemical evaluation of lozenges containing Lactobacillus reuteri as an adjunct to non-surgical periodontal therapy in chronic periodontitis. J. Periodontol. 86, 746-754 (2015).

35. Schmalz, G. et al. MMP-8 and TIMP-1 are associated to periodontal inflammation in patients with rheumatoid arthritis under methotrexate immunosuppression first results of a cross-sectional study. J. Microbiol. Immunol. Infect. 52, 386-394 (2019).

36. Ayravainen, L. et al. Anti-rheumatic medication and salivary MMP-8, a biomarker for periodontal disease. Oral. Dis. 24, 1562-1571 (2018).

37. Teixeira, M. K. S. et al. The modulation of the TREM-1/PGLYRP1/MMP-8 axis in peri-implant diseases. Clin. Oral. Investig. 24, 1837-1844 (2020).

38. Graziani, F. et al. Systemic inflammation after third molar removal: a case-control study. J. Dent. Res. 96, 1505-1512 (2017).

39. Camacho-Alonso, F. et al. Salivary myeloperoxidase and malondialdehyde are increased in patients exhibiting an asymptomatic mandibular impacted third molar. Med. Oral. Patol. Oral. Cir. Bucal 24, e537-e544 (2019).

40. Camelo-Castillo, A. et al. Relationship between periodontitis-associated subgingival microbiota and clinical inflammation by $16 \mathrm{~S}$ pyrosequencing. J. Clin. Periodontol. 42, 1074-1082 (2015).

41. Chen, W. P. et al. Composition analysis and feature selection of the oral microbiota associated with periodontal disease. Biomed. Res. Int. 2018, 3130607 (2018).

42. Shen, L. H., Xiao, E., Wang, E. B., Zheng, H. \& Zhang, Y. High-throughput sequencing analysis of microbial profiles in the dry socket. J. Oral. Maxillofac. Surg. 77, 1548-1556 (2019).

43. Menon, R. K. et al. Long-term impact of oral surgery with or without amoxicillin on the oral microbiome-a prospective cohort study. Sci. Rep. 9, 18761 (2019).
44. Shi, M. et al. The subgingival microbiome of periodontal pockets with different probing depths in chronic and aggressive periodontitis: a pilot study. Front. Cell Infect. Microbiol. 8, 124 (2018).

45. Verma, D., Garg, P. K. \& Dubey, A. K. Insights into the human oral microbiome. Arch. Microbiol. 200, 525-540 (2018).

46. Zaura, E., Keijser, B. J., Huse, S. M. \& Crielaard, W. Defining the healthy "core microbiome" of oral microbial communities. BMC Microbiol. 9, 259 (2009).

47. Arredondo, A., Blanc, V., Mor, C., Nart, J. \& Leon, R. Azithromycin and erythromycin susceptibility and macrolide resistance genes in Prevotella from patients with periodontal disease. Oral. Dis. 25, 860-867 (2019).

48. Koychev, S., Dommisch, H., Chen, H. \& Pischon, N. Antimicrobial effects of mastic extract against oral and periodontal pathogens. J. Periodontol. 88, 511-517 (2017).

49. Perez-Chaparro, P. J. et al. Newly identified pathogens associated with periodontitis: a systematic review. J. Dent. Res. 93, 846-858 (2014).

50. Socransky, S. S., Haffajee, A. D., Cugini, M. A., Smith, C. \& Kent, R. L. Jr Microbial complexes in subgingival plaque. J. Clin. Periodontol. 25, 134-144 (1998).

51. Belstrom, D. et al. Influence of periodontal treatment on subgingival and salivary microbiotas. J. Periodontol. 89, 531-539 (2018).

52. Liu, G. et al. Shift in the subgingival microbiome following scaling and root planing in generalized aggressive periodontitis. J. Clin. Periodontol. 45, 440-452 (2018).

53. Ikeda, E. et al. Deep sequencing reveals specific bacterial signatures in the subgingival microbiota of healthy subjects. Clin. Oral. Investig. 23, 1489-1493 (2019).

54. Correa, F. O. B., Goncalves, D., Figueredo, C. M. S., Gustafsson, A. \& Orrico, S. R. P. The short-term effectiveness of non-surgical treatment in reducing levels of interleukin-1beta and proteases in gingival crevicular fluid from patients with type 2 diabetes mellitus and chronic periodontitis. J. Periodontol. 79, 2143-2150 (2008).

\section{(i)}

Open Access This article is licensed under a Creative Commons Attribution 4.0 International License, which permits use, sharing, adaptation, distribution and reproduction in any medium or format, as long as you give appropriate credit to the original author(s) and the source, provide a link to the Creative Commons license, and indicate if changes were made. The images or other third party material in this article are included in the article's Creative Commons license, unless indicated otherwise in a credit line to the material. If material is not included in the article's Creative Commons license and your intended use is not permitted by statutory regulation or exceeds the permitted use, you will need to obtain permission directly from the copyright holder. To view a copy of this license, visit http://creativecommons. org/licenses/by/4.0/.

(c) The Author(s) 2021 